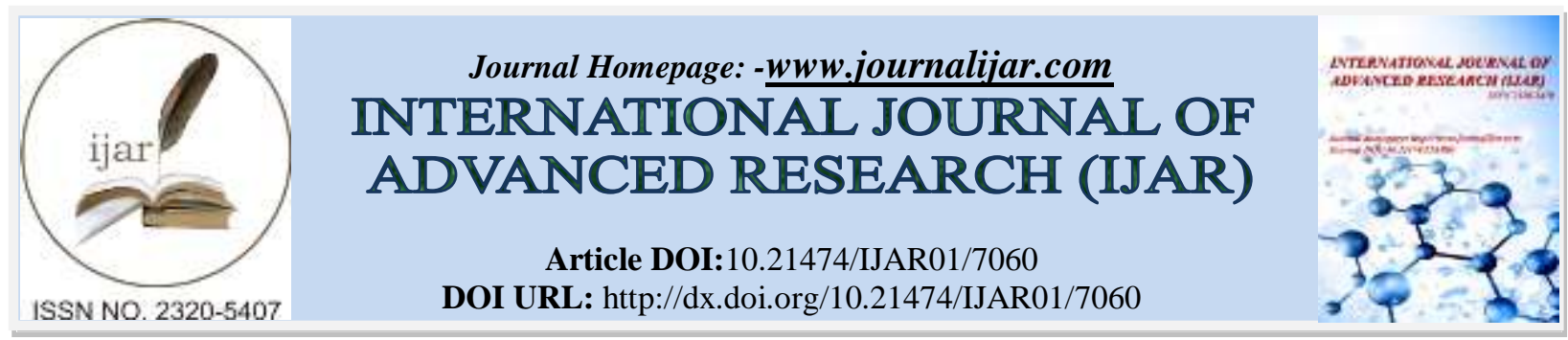

RESEARCH ARTICLE

\title{
IMPACTED MANDIBULAR DECIDUOUS AND PERMANENT CANINES: A 'DOUBLE TROUBLE' IN THE PATHOGENESIS OF ADENOMATOID ODONTOGENIC TUMOR: A CASE REPORT AND REVIEW OF LITERATURE.
}

Dr. Viola Rodrigues ${ }^{1}$, Dr. Anita Spadigam ${ }^{2}$, Dr. Anita Dhupar ${ }^{3}$ and Dr. Shaheen Syed ${ }^{4}$.

1. Post graduate student, Department of Oral Pathology and Microbiology, Goa Dental College and Hospital, Bambolim-Goa.

2. Professor and Head, Department of Oral Pathology and Microbiology, Goa Dental College and Hospital, Bambolim-Goa.

3. Professor, Department of Oral Pathology and Microbiology, Goa Dental College and Hospital, Bambolim-Goa.

4. Assistant Professor Department of Oral Pathology and Microbiology, Goa Dental College and Hospital, Bambolim-Goa.

\section{Manuscript Info}

Manuscript History

Received: 08 March 2018

Final Accepted: 10 April 2018

Published: May 2018

Keywords:-

Adenomatoid Odontogenic Tumor,

Hamartoma, Neoplasm.

\section{Abstract}

Adenomatoid odontogenic tumor (AOT) is a benign non-invasive odontogenic lesion with slow growth potential and exceptionally low recurrence rate. This case report highlights an unusual case of an aggressive adenomatoid odontogenic tumor associated with impacted deciduous and permanent canines presenting in a 14 year old male in the anterior mandible.

Copy Right, IJAR, 2018,. All rights reserved.

\section{Introduction:-}

Odontogenic tumors represent a spectrum of lesions ranging from malignant (rare) and benign neoplasms to dental hamartomas, all arising from odontogenic residues, i.e. odontogenic epithelia and/or ectomesenchyme with variable amounts of dental hard tissues formed generally in the same sequence as in normal tooth development.[1]

The WHO histological typing of odontogenic tumors, jaw cyst and allied lesions (2005) has defined Adenomatoid odontogenic tumor (AOT) as "a tumor composed of odontogenic epithelium in a variety of histoarchitectural patterns, embedded in a mature connective tissue stroma and characterized by slow but progressive growth". [2]

AOT was first termed as 'epithelioma adamantinum' by Steensland (1905), followed by Dreibladt (1907) as a 'pseudo adenoameloblastoma'. It was later redesignated the term 'Adenomatoid odontogenic tumor' by Philipsen and Birn (1969) and represents 3-7\% of all odontogenic tumors. [3-5]

This tumor has sometimes been referred to as tumor of "two-third," because two-third of them occur in young females, two-third in the maxilla, two-third are associated with unerupted teeth, two-third are associated with canines and two-third of the tumors are diagnosed in the second decade of life.[5,6]

It has three well recognized clinicopathological variants- intrafollicular, extrafollicular and peripheral with 20 recognized histopathological variations. $[4,6,7]$ 


\section{Case report:-}

A 14 year old male reported to the Goa Dental College and Hospital for evaluation of a gradually increasing swelling in relation to right lower jaw since two months.

Extra oral examination revealed a single, diffuse firm and non-tender swelling in relation to the right parasymphysis region measuring approximately $4 \times 3 \mathrm{cms}$. Intra oral examination revealed a diffuse swelling in relation to 42,44 , 45 , and 46 obliterating the buccal vestibule with expansion of lingual and buccal cortical plates. The swelling was hard, non- tender, non- pulsatile and non- fluctuant. The teeth in the area, 44, 45, and 46 tested non-vital. Orthopantomograph revealed a unilocular radiolucency crossing the midline and extending from 32 to 47 , oval in shape with well-defined borders. Impacted deciduous and permanent canines were seen within the radiolucent lesion with radio-opaque flecks and resorption of the roots of overlying teeth. [Figure 1]

A provisional diagnosis of Adenomatoid odontogenic tumor was given.

Surgical excision of mass was followed by histopathological evaluation. On gross examination, the cut surface revealed granular hard tissue in relation to the crown of 43 .

$\mathrm{H} / \mathrm{E}$ stained sections showed odontogenic epithelium arranged in varied histoarchitectural patterns of ductules, rosettes, cell nests, sworls and sheets with a very scant connective tissue stroma surrounded by a fibrous capsule. Ductules and rosettes were lined by a single layer and double layer of cuboidal to low columnar cells respectively with vesicular hyperchromatic nuclei polarized towards the basement membrane. Centre of the ductules were clear, other areas showed eosinophilic coagulum. Nests and sheets of spindle to cuboidal cells were seen. Dystrophic calcifications in the form of spherical, ovoid and linear hematoxyphilic masses, some as Liesegang rings were seen.

[Figure 2]

\begin{tabular}{|c|c|}
\hline Research supporting hamartomatous nature & Research supporting neoplastic nature \\
\hline $\begin{array}{l}\text { Similar immunohistochemical composition of AOT with } \\
\text { respect to reduced enamel epithelium and enamel organ } \\
\text { (Amelogenin, enamelin, sheathelin, enamelysin) } \\
\text { Ameloblastoma has greater proliferative potential than } \\
\text { AOT } \\
\text { Low Ki-67 expression } \\
\text { No } 16 \text { expression } \\
\text { More invasive behaviour of ameloblastomas compared } \\
\text { to AOT } \\
\text { Higher percentage of Ki-67 and Bcl-2 in solid } \\
\text { ameloblastoma compared to AOT }\end{array}$ & $\begin{array}{l}\text { Most odontogenic tumors are monoclonal } \\
\text { Marked expression of cyclin D1 in AOT } \\
\text { Spindle-shaped tumor cells of AOT show close } \\
\text { associations with extracellular matrix signaling as well } \\
\text { as cell proliferation } \\
\text { Strong immunolocalization of HGF (hepatocyte growth } \\
\text { factor and c-met in squamous cells present in AOT's } \\
\text { Strong cytoplasmic expression of } \beta \text {-catenin } \\
\text { Similar proliferative potential between ameloblastoma } \\
\text { and AOT }\end{array}$ \\
\hline
\end{tabular}

\begin{tabular}{|l|l|l|}
\hline Features & Typical findings of AOT & Unusual features present in our case \\
\hline Sex & Females & Male \\
\hline Site & Anterior maxilla & Anterior mandible \\
\hline Size & Does not exceed 1-3cm & 4 x $3 \mathrm{cms}$ \\
\hline Root resorption & Extremely rare & Seen \\
\hline Cortical plate penetration & Rare & Seen (lingual) \\
\hline Impacted tooth & Usually one & Deciduous and permanent mandibular canines \\
\hline \multicolumn{2}{|l|}{ Table II:-Unusual findings seen in our case related to typical features of an AOT } \\
\hline
\end{tabular}




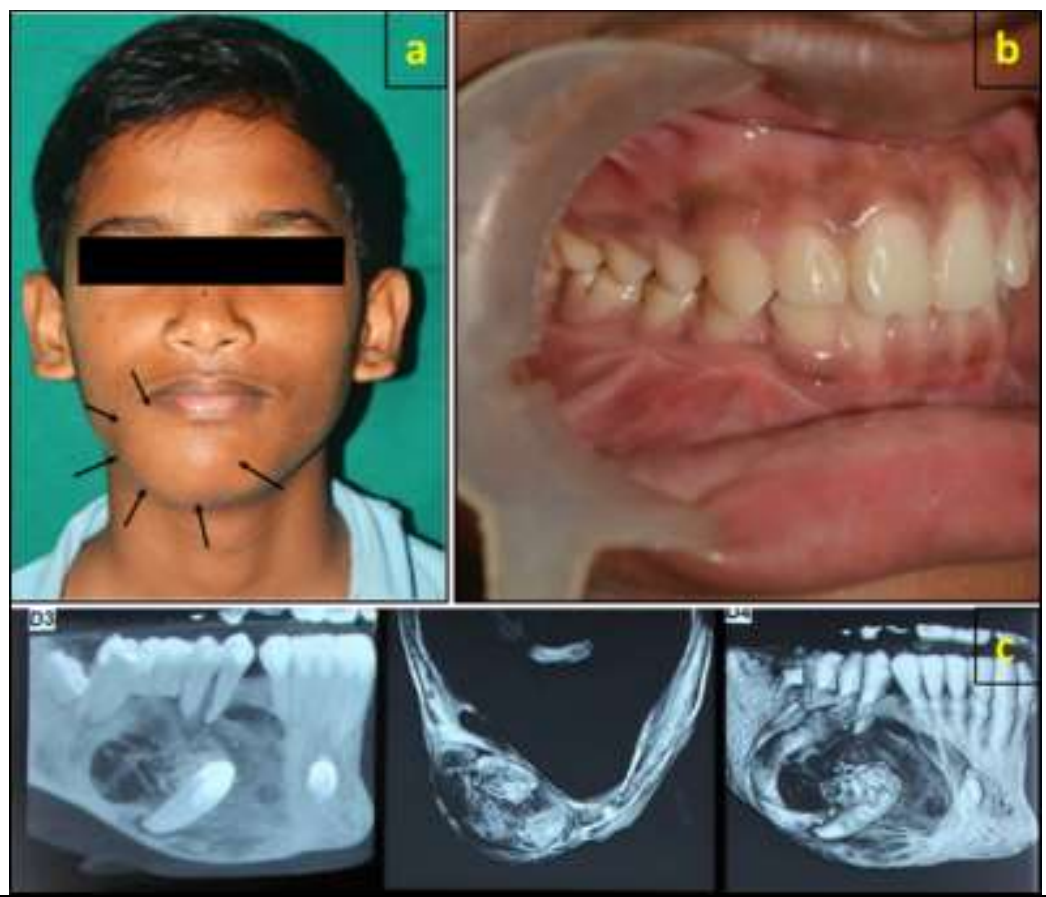

Figure 1:-a) Swelling in relation to the right parasymphysis region. b) Swelling in relation to 42, 44, 45, and 46 obliterating the buccal vestibule. c) Impacted deciduous and permanent canines seen within the radiolucent lesion with radio-opaque flecks in relation to the crown of the permanent canine and resorption of the roots of overlying teeth.

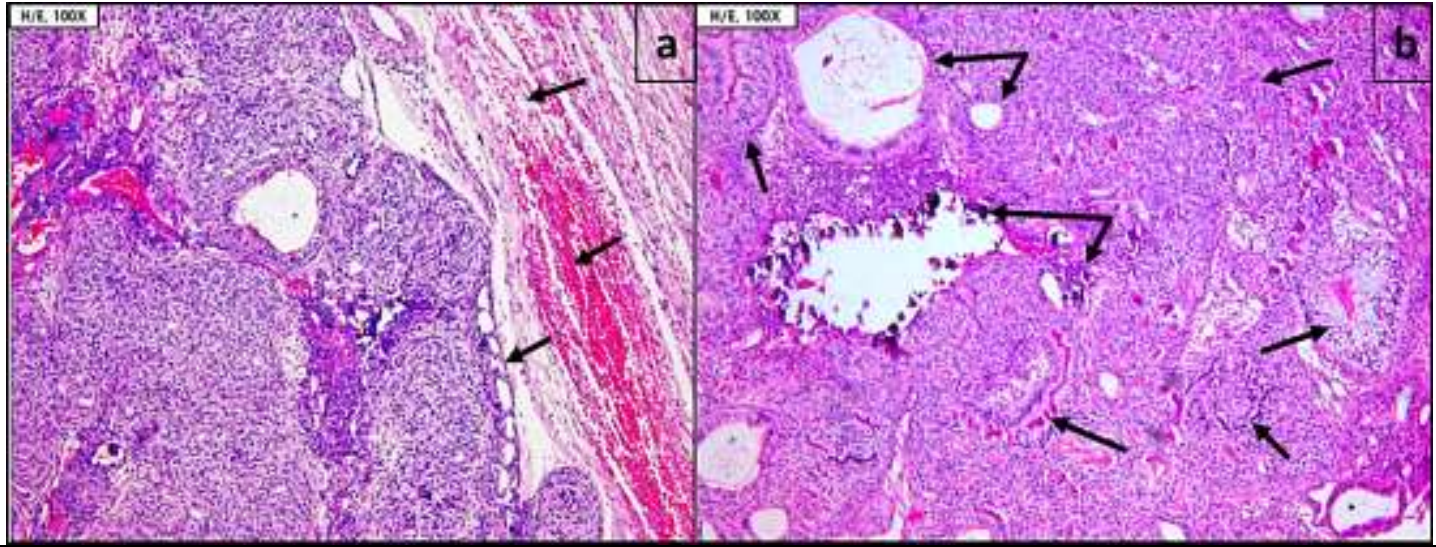

Figure 2:-a and b) Photomicrograph showing ductules, rosettes, cell nests, sworls and sheets of odontogenic epithelium with a very scant connective tissue stroma surrounded by a fibrous capsule.

\section{Discussion:-}

The Adenomatoid Odontogenic Tumor (AOT) is a benign (hamartomatous) non-invasive odontogenic lesion with slow growth potential and exceptionally low recurrence rate.

AOT's are said to arise from dental lamina remnants in the gubernacular cord of a developing permanent incisor, canine or premolar (96\% cases) and from the epithelial lining of odontogenic cysts. [8, 9]

AOT's have 3 distinct clinical and radiographic variants: (a) Central (or intra-osseous) variant (b) Extra-follicular (or extra-coronal) variant and (c) Peripheral (or gingival) variant (PAOT): (i) 'peripheral AOT variant' (ii) 'erupted intra-osseous PAOT variant'. [4,6] 
There is an ongoing debate whether AOT is a hamartoma or a true neoplasm. [Table I]

Immunohistochemically, the classical AOT phenotype is characterized by a cytokeratin (CK) profile similar to follicular cysts and/or oral or gingival epithelium based on positive staining with CK5, CK17 and CK19. [11, 12]

Conservative surgical enucleation is the treatment modality of choice. [3]

Eighteen cases of mandibular AOT's in male patients have been reported in literature. Only one case with two impacted teeth has been reported in a female patient in anterior mandible. [6, 13-20] [Table II]

Recurrence of AOT is exceptionally rare and therefore the prognosis is excellent.[5] But in cases where unusual findings are seen, the follow up period should be extended to check for recurrences, as seen in the present case.

\section{Conclusion:-}

AOT is an uncommon odontogenic lesion, which can be readily identified from its histopathological features. Impacted deciduous teeth and unerupted succeeding permanent teeth, when associated with a swelling, always need to be investigated for odontogenic pathoses.

Financial support and sponsorship:- Nil

Conflict of Interest:- There were no conflicts of interest.

\section{References:-}

1. William G. Shafer, Maynard K. Hine, Barnet M. Levy; Shafer's Textbook of Oral Pathology; 8th EditionElsevier.

2. Barnes L, Eveson JW, Reichart PA, Sidransky D. World Health Organization classification of tumours: Pathology and Genetics of tumours of the head and neck; Lyon: IARC; 2005. pp. 304-5

3. Namrata N Patil, Abhishek Singh Nayyar, Vijay Wadhwan; Adenomatoid odontogenic tumor: A series of four clinico-pathological variants; International Journal of Case Reports and Images 2014; (1):1-7.

4. Hans Peter Philipsen, Pathawee Khongkhunthiang, Peter A. Reichart; The adenomatoid odontogenic tumour: an update of selected issues; J Oral Pathol Med (2016).

5. Sethi S, Kumar M, Aggarwal P, Indra Kumar HS, Sugandhi CD, Singh S. A case report and short review on changing trends in the site of occurrence of adenomatoid odontogenic tumor: Unravelling the past 15 years. Dent Res J 2016; 13: 462-71.

6. Manoj Kumar Jain and Sheetal Oswal K, Adenomatoid Odontogenic Tumor of Mandible - 'Master of Disguise'; J Dent App. 2014;1(3): 40-42

7. Vimi S Mutalik, Ashish Shreshtha, Sunil S Mutalik, and Raghu Radhakrishnan; Adenomatoid odontogenic tumor: A unique report with histological diversity; J Oral MaxillofacPathol. 2012 Jan-Apr; 16(1): 118-121.

8. Fumio Ide, Kenji Mishima, Kentaro Kikuchi et al; Development and growth of adenomatoid odontogenic tumor related to formation and eruption of teeth; Head and Neck Pathol (2011) 5:123-132

9. Philipsen HP. Samman N. Ormiston IW. Wu PC, Reichert PA: Variants of the adenomatoid odontogenic tumor with a note on tumor origin. J Oral Pathol Med 1992; 21; 34852.

10. Patil S, Rao RS, Majumdar B. Hamartomas of the oral cavity. J Int Soc Prevent Communit Dent 2015;5: 34753.

11. Marcelo Macedo Crivelini, Ana Maria Pires Soubhia, Renata Callestini Felipini; Study on the origin and nature of the adenomatoid odontogenic tumor by immunohistochemistry; J Appl Oral Sci. 2005;13(4):406-12.

12. Praveen Anigol, Venkatesh V. Kamath, Krishnanand Satelur, Nagaraja Anand, Komali Yerlagudda; Amelogenin in odontogenic cysts and tumors: An immunohistochemical study; National Journal of Maxillofacial Surgery, Vol 5, Issue 2, Jul-Dec 2014.

13. Vikas Singh, D J Bhaskar, R Chandan Agali et al; Adenomatoid odontogenic tumour: Report of a case and review of Literature; International Journal of Scientific Study January 2014 Vol 1 Issue 4.

14. Jörg GK Handschel, Rita A Depprich, André C Zimmermann, Stefan Braunstein and Norbert R Kübler; Adenomatoid odontogenic tumor of the mandible: review of the literature and report of a rare case; Head \& Face Medicine 2005, 1:3. 
15. Ramandeep Saluja, Gurkiran Kaur, and Preetinder Singh; Aggressive adenomatoid odontogenic tumor of mandible showing root resorption: A histological case report; Dent Res J (Isfahan). 2013 Mar-Apr; 10(2): 279282.

16. Chandramani B. More, Sunanda Das, Swati Gupta, and Khushbu Bhavsar; Mandibular adenomatoid odontogenic tumor: Radiographic and pathologic correlation; J Nat Sci Biol Med. 2013 Jul-Dec; 4(2): 457-462.

17. Sunil S. Mishra, Shirish S. Degwekar, Mukta B. Motwani, Ruchi M. Anand; Adenomatoid odontogenic tumor with impacted mandibular canine: a case report; J Clin Exp Dent. 2011;3(Suppl1):e373-6.

18. UI Belgaumi, MI Parkar et al; Follicular Adenomatoid Odontogenic Tumor in Mandible: A Rare Case Report; Ann Med Health Sci Res. 2015 Nov-Dec; 5(6): 469-472.

19. Ramandeep Salujaet al, Aggressive adenomatoid odontogenic tumor of mandible showing root resorption: A histological case report; Dent Res J (Isfahan). 2013 Mar-Apr; 10(2): 279-282.

20. T.G. Shrihari, adenomatoid odontogenic tumor with rare clinical and radiological presentation- a case report; Pacific Journal of Medical Sciences: Vol. 9, No. 1, Sept 2011. 